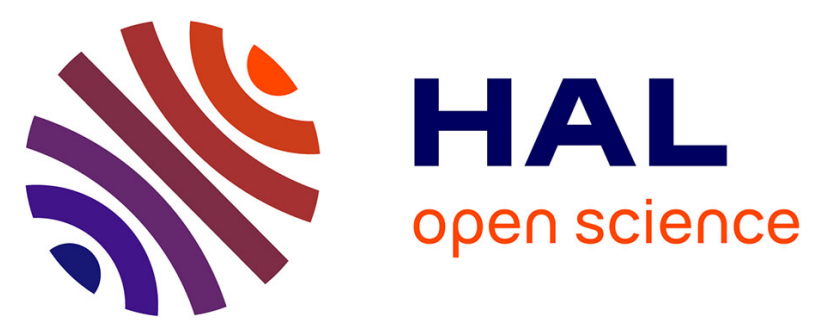

\title{
Layer-by-Layer Enzymatic Platform for Stretched-Induced Reactive Release
}

Cédric Vogt, Damien Mertz, Karim Benmlih, Joseph Hemmerlé, Jean-Claude Voegel, Pierre Schaaf, Philippe Lavalle

\section{- To cite this version:}

Cédric Vogt, Damien Mertz, Karim Benmlih, Joseph Hemmerlé, Jean-Claude Voegel, et al.. Layerby-Layer Enzymatic Platform for Stretched-Induced Reactive Release. ACS Macro Letters, 2012, 1 (7), pp.797-801. 10.1021/mz3000896 . hal-03101026

\section{HAL Id: hal-03101026 \\ https://hal.science/hal-03101026}

Submitted on 6 Jan 2021

HAL is a multi-disciplinary open access archive for the deposit and dissemination of scientific research documents, whether they are published or not. The documents may come from teaching and research institutions in France or abroad, or from public or private research centers.
L'archive ouverte pluridisciplinaire HAL, est destinée au dépôt et à la diffusion de documents scientifiques de niveau recherche, publiés ou non, émanant des établissements d'enseignement et de recherche français ou étrangers, des laboratoires publics ou privés. 


\title{
Layer-by-layer enzymatic platform for stretched-induced reac- tive release
}

\author{
Cédric Vogt ${ }^{1,2}$, Damien Mertz ${ }^{1,2}$, Karim Benmlih ${ }^{1,2}$, Joseph Hemmerlé ${ }^{1,2}$, Jean-Claude Voegel ${ }^{1,2}$, Pierre \\ Schaaf ${ }^{\beta^{*}}$, Philippe Lavalle ${ }^{1,2,4}$
}

1) Institut National de la Santé et de la Recherche Médicale, INSERM Unité 977, "Biomaterials and Tissue Engineering",11 rue Humann, 67085 Strasbourg Cedex, France

2) Faculté de Chirurgie Dentaire, Université de Strasbourg, 1 Place de l'Hôpital, 67000 Strasbourg, France

3) Centre National de la Recherche Scientifique, UPR22, Institut Charles Sadron, 23 rue du Loess, BP 84047, 67034 Strasbourg, Cedex 2, France

4) Hôpitaux Universitaires de Strasbourg, 1 Place de l'Hôpital, 67000 Strasbourg, France

\begin{abstract}
An original "all-in-one" platform combining polymers, enzymes and substrates in a unique film is designed. A polymeric barrier strata prevents any contact between enzymes adsorbed on top of the film and substrates loaded in an underlying reservoir. Upon stretching of the film, a continuous diffusion of the substrates through the barrier is triggered, followed by a catalytic reaction. This leads to the formation of products that are released in solution. This new platform acts as a stretch-induced reactive release system and emerges as an innovative concept in mechano-responsive materials.
\end{abstract}

The design of responsive materials emerges as an extremely hot topic. In particular mechanosensitive materials are now thoroughly investigated. ${ }^{1}$ Nanosciences and nanotechnologies allowed recently the development of devices and the implementation of tools to detect a single molecular event that originates from a mechanical stimulus. For example, mechanochromic materials, i.e. materials that change colour when undergoing a tensile stress, have been designed and allow to directly visualize a mechanochemical reaction. ${ }^{2-7}$ Another recent application depicts self-reporting hybrid materials where proteins are used as reporter molecules that sense deformation, strain, or mechanical damage in materials. ${ }^{8}$ Such mechanical sensitive architectures mimic the natural mechanism involved in mechanotransduction, where cells convert a mechanical stimulus into a cascade of chemical reactions. ${ }^{9}$

Mimicking nature and designing surfaces that would induce chemical reactions or trigger cellular responses upon stretching the surface is not only of fundamental interest but could also present numerous potentialities from a technological point of view. Such surfaces could be used, for example, to build sensors that become active or are regenerated under stretching, to control reactions in microfluidic devices upon local stretching of the substrate or to induce specific biological reactions on cells seeded on such devices. A first step, based on polyelectrolyte multilayer films ${ }^{10}$, was recently undertaken by our group to design a "mechanotransductive" surface. ${ }^{11}$ For this purpose, a film combining two strata of polyelectrolyte multilayers ${ }^{12}$ was built: a first thick and hydrated stratum acting as a reservoir for enzymes was capped by a second thin and dense stratum which plays the role of barrier. In the unstretched state, enzymes are masked and the catalytic reaction is "off". Once a critical stretching degree is applied on the system, biocatalysis turns "on" at the film/solution interface due to the unmasking of enzymes. However, this system suffers from one drawback: a feedback inhibition process takes place during the catalysis and thus only a burst in substrate production could be monitored instead of a continuous enzymatic rate. Moreover, it was not possible to control the release of products through mechanical stimuli with this system.

The effect of mechanical stimuli on the release of compounds from polymeric matrices is a subject of major interest which is the focus of numerous studies. Lee et al. describes the control of growth factor release from alginate hydrogels with cyclic compression loadings. ${ }^{13,14}$ Due to these mechanical stresses, neovascularisation in tissue surrounding stimulated hydrogels can be enhanced. More recently, it has been demonstrated that the release of macromolecules from layer-by-layer capsules is strongly improved by compression. ${ }^{15}$ However to our knowledge, no mechanoresponsive device releasing products through a chemical process activated by stretching has yet been reported. Herein we address the design of an original "all-in-one" platform combining polymers, enzymes and substrates in a unique film. At rest, the catalysis is off due to a polymeric barrier that prevents any contact between enzymes and substrates. Upon stretching, embedded substrates diffuse through the barrier. Once reaching the anchored enzymes, reaction takes place and the products issue from the catalytic reaction are released in solution. This approach is strongly innovative in comparison to our previous studies devoted to the design of mechanotransductive surfaces $^{11}$ : in the previous system, the film was designed as an impermeable catalytic surface converting substrates in solution into products. In the present study, the substrate is embedded in the film and upon stretch the system converts it into product via enzymatic catalysis and releases it in solution. This new platform provides significant benefits: i) all the constituents i.e. substrates and enzymes are simultaneously present in the film; ii) mechanical stretches allow to control releases of molecules; iii) mechanical stimuli trigger a "time-controllable" kinetic of release and not a burst in a catalytic reaction; iv) no more feedback inhibition occurs 
in the catalytic reaction due to accumulation of substrate in the vicinity of enzymes. These two last points were the major drawbacks from our previous system. We expect the stretch-induced reactive release approach to be of interest because of the practical advantages it may provide. A macroscopic stretch is a simple stimulus from a technical point of view but we demonstrate it allows to control nanosystems in a precise manner. Moreover, the triggering of chemical reactions inducing release of processed-products instead of a direct release from the material also brings advantage to avoid storage of these products under their native form within the device but in a precursor form that can protect them from degradation for example.

In the present study, we used thick and hydrated exponentially growing polyelectrolyte multilayers, namely poly(Llysine)/hyaluronic acid (PLL/HA), to embed fluorescein diphosphate (FDP). The FDP molecule is a fluorogenic substrate of alkaline phophatase enzyme, i.e. it becomes strongly fluorescent once the enzyme has catalyzed the dephosphorylation reaction of FDP into fluorescein (F). FDP molecules deposited on top of a thick PLL/HA film diffuse inside and are concentrated within it. This film plays the role of reservoir for FDP as displayed by confocal microscopy images (Supporting Information Figure S1). Following FDP deposition, a capping constituted by (PDADMA/PSS) 10 (10 bilayers of PDADMA: poly(diallyldimethylammonium) / PSS: poly(sodium 4-styrenesulphonate)) strata was added on the top. The thickness was estimated to be around $90 \mathrm{~nm}$ from quartz crystal microbalance measurements (QCM) (Supporting Information Figure S2). It is well known that PDADMA/PSS layers act as a barrier and prevent diffusion of polyelectrolytes ${ }^{16}$ or proteins ${ }^{11}$ through it. In this study, PDADMA/PSS layers isolate FDP from the alkaline phosphatase enzymes (ALP). To validate the efficiency of this barrier, rhodamine-labeled ALP (ALPRho, red emission) was adsorbed as a last layer on top of the PDADMA/PSS layers that cap the PLL/HA film containing FDP. Despite the negative charges of the film induced by PSS chains adsorbed as final layer, ALPRho, also negatively charged, adsorbs significantly on the film according to confocal microscopy image (Supporting Information Figure $\mathrm{S}^{\prime} b^{\prime}$ ) and quartz crystal microbalance measurements (Supporting Information Figure S2a). If $\mathrm{ALP}^{\text {Rho }}$ is deposited on a similar film but with a PDADMA terminating layer instead of PSS, the whole film section displays red fluorescence (Supporting Information Figure S3). In such a case, the adsorbed amount of ALP is five times larger on PDADMA than on PSS, as checked with QCM (Supporting Information Figure S2b). However, the PDADMA/PSS capping layer becomes no more efficient as a barrier towards enzymes and the catalytic reaction occurs directly in the film even in the non-stretched state. This underlines the sensitivity of the barrier on the nature of the last deposited polyelectrolyte.

Deposition of ALP on top of the film shows no difference in the background level of green fluorescence intensities and demonstrates that no biocatalysis occurs (Supporting Information Figures S1a and S1b). Moreover, no evolution with time of the green fluorescence within the film and in the supernatant was monitored. This clearly indicates that the enzymatic substrate FDP and the enzyme ALP are both present in the film but in two distinct areas and that this "all-in-one" film $\left[(\mathrm{PLL} / \mathrm{HA})_{30} / \mathrm{PLL} / \mathrm{FDP} /(\mathrm{PDADMA} / \mathrm{PSS})_{10} / \mathrm{ALP}^{\mathrm{Rho}}\right]$ depicts no catalytic activity at rest. Finally, the film $(\mathrm{PLL} / \mathrm{HA})_{30} / \mathrm{PLL} / \mathrm{FDP} /(\mathrm{PDADMA} / \mathrm{PSS})_{10} / \mathrm{ALP}^{\text {Rho }}$ constitutes our "enzymatic platform" (Figure 1, step a) that will be selected all along the present study.
In order to check if a stress applied on the film can trigger the catalytic reaction and release the products of the enzymatic reaction, evolution with time of the fluorescence intensity in the supernatant was monitored with confocal microscope for stretching degrees $\alpha$ ranging between 0 and 100\% (Figure 2). For $\alpha$ values ranging between 0 and $40 \%$, a slight increase of the fluorescence intensity was monitored, i.e. about $0.10 \pm 0.05$ a.u. $s^{1}$. This value probably corresponds to a background level as attested by the slope of the same order obtained with a similar film but without enzymes on the top $(0.13$ a.u. s $\left.{ }^{1}\right)$. This rate seems also independent of the stretching degree (Supporting Information Figure S4).
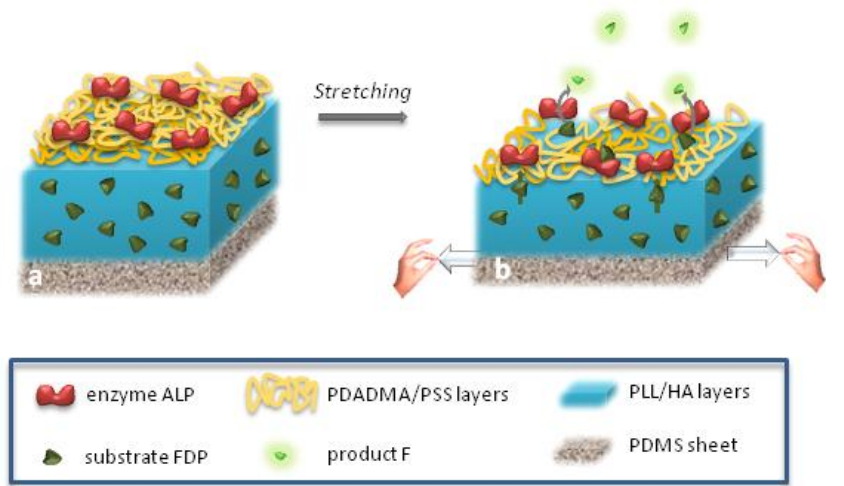

Figure 1. Schematic illustration of the mechanism involved in the enzymatic platform during stretching: a) before the stretching step and b) during the stretching step.

For $\alpha=60 \%$, the slope strongly increases, reaching about $0.80 \pm 0.20$ a.u. $s^{-1}$. This corresponds to a critical stretching degree $(\alpha=60 \%)$ at which the production of fluorescein molecules is triggered. For higher stretching degrees, the slope corresponding to the increase of fluorescein solution does not change any more. No significant further increase of the fluorescein production rate takes place beyond.

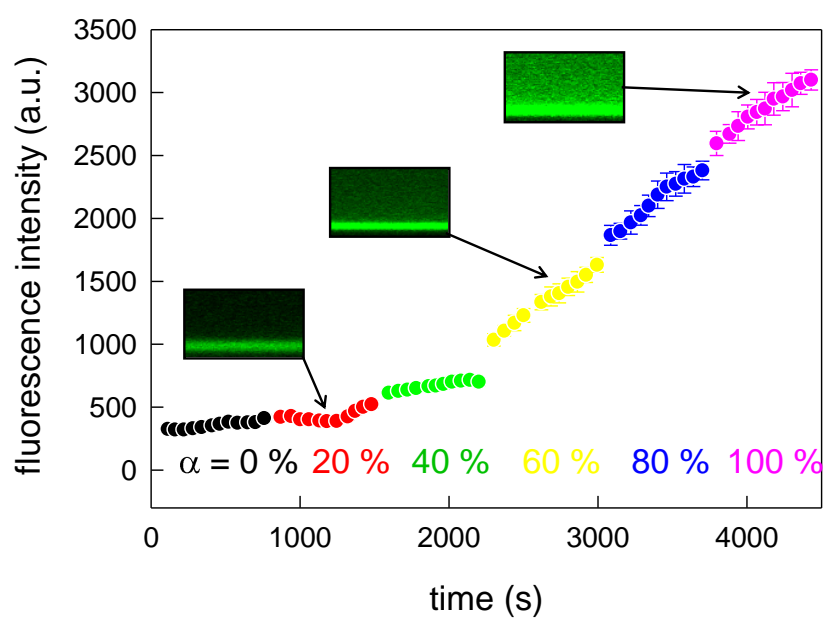

Figure 2. Evolution with time of mean fluorescence intensity of buffer solution in contact with the enzymatic platform. Several stretching degrees $\alpha$ were applied on the film: from $\alpha=0 \%$ to $\alpha=100 \%$. The typical stretching time from one stretching degree to the next was of the order of $15 \mathrm{~s}$. The film was then kept in a fixed stretched state $(\alpha=20 \%, 40 \%, 60 \%, 80 \%$ or $100 \%)$ for more than $1500 \mathrm{~s}$. The time steps between two consecutive fluores- 
cence measurements was $60 \mathrm{~s}$. The inserts correspond to confocal microscope section images $\mathrm{x}, \mathrm{z}\left(130 \times 76 \mu \mathrm{m}^{2}\right)$ acquired at $\mathrm{t}=1200 \mathrm{~s}$, $t=2800 \mathrm{~s}$ and $\mathrm{t}=4300 \mathrm{~s}$. The error bars correspond to standard deviations calculated from image analyses.

Inserts in Figure 2 shows confocal microscope section images before and after stretching the platform. An increase of fluorescence occurs both in the supernatant and in the film. This indicates that fluorescein molecules produced by the enzymes on top of the film diffuse within the underlying PLL/HA reservoir and are also continuously released into the supernatant. It should be noted that we never observed delamination or surface defects in the stretched films.

At this stage, enzymatic reaction rate is constant with time over at least 30 minutes, thus no feedback inhibition of the enzyme takes place in contrary to the previous designed systems. ${ }^{11}$ In the present study, the products of the reaction and more particularly phosphate ions are probably released in the supernatant and are not confined close to the enzymes.

To test the reversibility of the system, the enzymatic platform was stretched at $\alpha=100 \%$ and brought back to $\alpha=0 \%$ (Supporting Information Figure S5). No change in the rate of fluorescein production was observed, the same slope was maintained once the system was brought back at rest. Thus, the "opening" of the barrier which occurs at stretch is not reversible. This permeability could originate from pores appearing in the barrier layer. However no pores were visualized on top of the film in the stretched state as demonstrated by AFM images (Supporting Information Figure S6). We characterized the electric surface potential before and after the stretching step and no strong variations were monitored ($2.44 \pm 0.13 \mathrm{mV}$ and $-4.18 \pm 0.59 \mathrm{mV}$ respectively). Moreover, no desorption of enzymes was monitored during the whole experiments, the red fluorescence corresponding to ALP ${ }^{\text {Rho }}$ adsorbed on top of the film remains unchanged with time, whatever the stretching degree $\alpha$. Finally, during the stretching step, the barrier probably becomes less dense permitting the FDP molecules to pass through it. When the film is brought back at rest, the barrier on top of the reservoir should be less structured compared to its initial state and efficiency towards diffusion of small molecules is lost.

A thicker barrier constituted with 20 PDADMA/PSS bilayers instead of 10 bilayers was also tested. This barrier remained tight when the system was stretched up to $140 \%$, indicating that no enzymatic reaction occurs (Supporting Information Figure S7). FDP molecules were in this case not able to cross the barrier, whatever the mechanical state of the film. For thinner barriers (2 or 6 PDADMA/PSS bilayers) the catalysis already occurs when the film is at rest (data not shown). This demonstrates that the barrier is too thin and not tight enough. Finally, the enzymatic platform constituted by 10 (PDAMDA/PSS) bilayers corresponds to an optimal design for our purpose, i.e. trigger a reactive release through a mechanical stimulus.

To elucidate the mechanism involved in the product release, the supernatants above stretched films with and without ALP adsorbed onto the barrier were analyzed by fluorometry. Fluorescence intensity of the supernatant of the enzymatic platform $\left[(\mathrm{PLL} / \mathrm{HA})_{30} / \mathrm{PLL} / \mathrm{FDP} /(\mathrm{PDADMA} / \mathrm{PSS})_{10} / \mathrm{ALP}\right.$ film] was measured $40 \mathrm{~min}$ after stretching the film at $100 \%$ (Figure 3, blue bar on the foreground). A control experiment with similar conditions but for a film without enzyme on top was also performed (Figure 3, grey bar on the foreground). As expected, the fluorescence of the supernatant for the control system is much lower compared to the enzymatic platform where the enzymes catalyze the production of a large amount of fluorescein molecules which are afterwards released in the supernatant. The non-zero fluorescence monitored for the control system is attributed to the few F molecules initially present in the commercial product and which are probably slowly released from the film upon stretch. In a further experiment, we added a large excess of ALP solution to both of these sampled supernatants (without any film in contact) and performed a second series of measurements (Figure 3, bars on the background). For our enzymatic platform, same fluorescence intensities were measured with or without addition of enzyme in the supernatant showing that no FDP but only the F molecules were released after stretching. This strongly suggests that all FDP molecules diffusing out of the film are dephosphorylated by the enzymes on top of the film and are released as F molecules (Figure 2, step b). Thus, the rate of diffusion of FDP molecules out of the film is lower than the enzymatic reaction rate. For the control system, fluorescence intensity of the supernatant strongly increases after enzyme addition and this intensity became identical to the enzymatic platform. This clearly demonstrates that when the film was stretched in the absence of adsorbed enzyme on the top, FDP was released from the film to the supernatant. This film being not coated with an enzyme layer, no dephosphorylation of FDP took place. We hypothesize that the barrier should undergo some reorganization under stretching which makes the barrier permeable towards FDP.

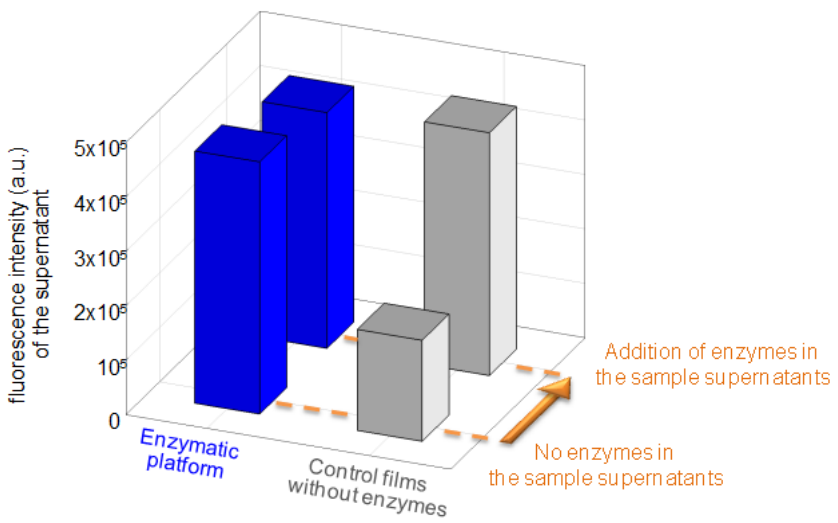

Figure 3. Fluorescence intensities of supernatants which were in contact with the enzymatic platform (blue) and a control film without enzyme. The supernatants were sampled after $40 \mathrm{~min}$ in the stretched state $(\alpha=100 \%)$ and fluorescence were measured with a fluorometer. Bars on the foreground correspond to a direct measurement of fluorescence and bars on the background to a measurement of the fluorescence after an addition of a large excess of ALP in the sample solution. The standard deviations for these measurements are below $5 \times 10^{4}$ a.u.

A new type of experiment was then designed to clarify the underlying mechanism: i) stretching the enzymatic platform at $\alpha=100 \%$ for $15 \mathrm{~min}$, ii) rinsing it for $5 \mathrm{~min}$ and iii) monitoring again fluorescence evolution of a fresh buffer solution brought in contact with the platform maintained in the stretched state (Figure 4a). After this rinsing step iii), the film being maintained at $\alpha=100 \%$, the fluorescence intensity recovered to a constant background level during ca. $15 \mathrm{~min}$. After this period, the release of fluorescein molecules starts again progressively and the fluorescence intensity rises up again with a high rate. The rinsing step at $\alpha=100 \%$ has probably induced a fast release of the FDP molecules located in the upper part of the reservoir (under the barrier) (Figure 4b). Thus, the remaining FDP molecules located more deeply in the film 
probably diffuse in the upper part and then are dephosphorylated by enzymes before to be released as fluorescein molecules in the supernatant. This mechanism is certainly fully diffusion-controlled. Finally, Figure 1 overviews the concept of stretch-induced reactive release. The designed and so-called "all in one enzymatic platform" allows to release catalyzed products from the film to the solution through a mechanical stimulus. Both enzymes and substrates are initially embedded in the polymeric architecture with no reaction occurring and a mechanical stress applied to the system is necessary to initiate the catalysis. This reaction takes place without any feedback inhibition process and leads to a release of the catalyzed products according to a linear profile with time. The mechanism behind this behavior corresponds to the diffusion of the substrate through the stretched film followed by its conversion into products by the adsorbed enzymes on top of the film. The products then diffuse in the solution.
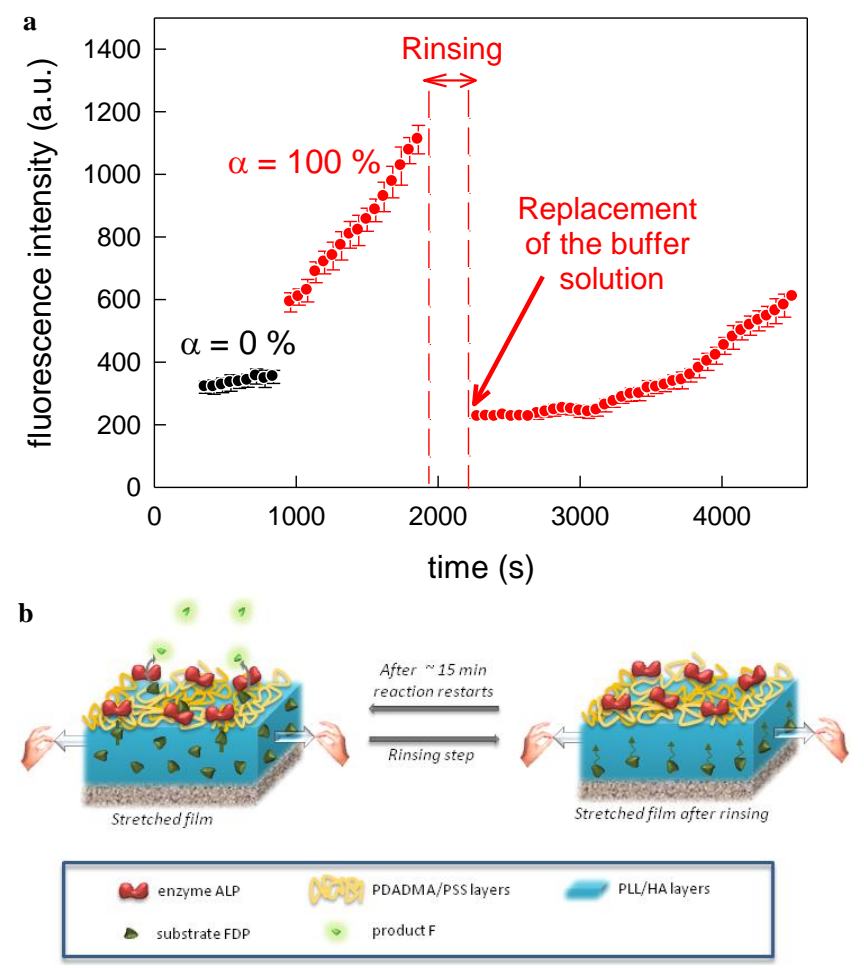

Figure 4. a) Evolution with time of mean fluorescence intensity of buffer solution in contact with the enzymatic platform. First, the enzymatic platform is at rest $(\alpha=0 \%$, black discs) and then a stretching is applied above the critical stretching degree $(\alpha=100 \%$, red discs). After ca. $15 \mathrm{~min}$, a rinsing step of $5 \mathrm{~min}$ is performed and supernatant is replaced by a fresh buffer solution. b) Schematic illustration of the mechanism involved in the enzymatic platform during stretching and after a rinsing step. The error bars correspond to standard deviations calculated from image analyses.

Finally, the designed platform represents a new concept in mechanotransductive materials which could find numerous applications in many fields and in particular in prodrug delivery. These prodrugs could be stored in a reservoir and converted through enzymatic reaction into drugs while crossing the enzymatic barrier.
Activation of this reactive release could be obtained by a local mechanical stimulus.

Supporting Information. Details of materials, experimental procedure, methods of characterization (confocal laser scanning microscope, quartz crystal microbalance, fluorometry, atomic force microscope, zeta potential measurements), confocal microscope, QCM and Zeta potential control experiments and AFM images. This material is available free of charge via the Internet at http://pubs.acs.org.

\section{Corresponding Author}

Prof. P. Schaaf, pierre.schaaf@ics-cnrs.unistra.fr

\section{Author Contributions}

The manuscript was written through contributions of all authors. All authors have given approval to the final version of the manuscript.

\section{Funding Sources}

This work was supported by Agence Nationale de la Recherche through "Programme Blanc" (project "BioStretch", ANR-10-BLAN0818). C.V. acknowledges the French Ministry of Education and Research for financial support. P.L. is indebted to Hôpitaux Universitaires de Strasbourg for financial support. P.S. acknowledges the International Center for Frontier Research in Chemistry for the grant "Enzymatically active materials reversible controllable by mechanical stretching".

\section{ACKNOWLEDGMENTS}

We thank J. Ortscheid for preliminary experiments, C. Ringwald for Zeta potential measurements and C. Bouthier for her assistance.

\section{ABBREVIATIONS}

PLL, Poly(L-lysine); HA, Hyaluronic acid; FDP, fluorescein diphosphate; F, Fluorescein; ALP, Alkaline phosphatase; ALP ${ }^{\text {Rho }}$, rhodamine-labeled Alkaline phosphatase; PDADMA, poly(diallyldimethylammonium); PSS, poly(sodium 4. styrenesulphonate); QCM, quartz crystal microbalance.

\section{REFERENCES}

(1) Ariga, K.; Mori, T.; Hill, J. P. Adv Mater 2012, 24, 158-176.

(2) Davis, D. A.; Hamilton, A.; Yang, J.; Cremar, L. D.; Van Gough, D.; Potisek, S. L.; Ong, M. T.; Braun, P. V.; Martinez, T. J.; White, S. R.; Moore, J. S.; Sottos, N. R. Nature 2009, 459, 68-72.

(3) Chung, C. M.; Cho, S. Y.; Kim, J. G. Sensor Actuat. B-Chem. 2008, 134, 822-825.

(4) Karthikeyan, S.; Sijbesma, R. P. Macromolecules 2009, 42, 5175-5178.

(5) Roberts, D. R. T.; Holder, S. J. J. Mater. Chem. 2011, 21, 82568268.

(6) Ramachandran, D.; Urban, M. W. J. Mater. Chem. 2011, 21, 8300-8308.

(7) Pucci, A.; Ruggeri, G. J. Mater. Chem. 2011, 21, 8282-8291.

(8) Bruns, N.; Pustelny, K.; Bergeron, L. M.; Whitehead, T. A.; Clark, D. S. Angew. Chem. Int. Ed. 2009, 48, 5666-5669.

(9) Katsumi, A.; Orr, A. W.; Tzima, E.; Schwartz, M. A. J. Biol. Chem. 2004, 279, 12001-12004. 
(10) Decher, G. Science 1997, 277, 1232-1237.

(11) Mertz, D.; Vogt, C.; Hemmerlé, J.; Mutterer, J.; Ball, V.; Voegel, J.-C.; Schaaf, P.; Lavalle, P. Nat. Mat. 2009, 8, 731-735.

(12) Garza, J. M.; Schaaf, P.; Muller, S.; Ball, V.; Stoltz, J. F.; Voegel, J. C.; Lavalle, P. Langmuir 2004, 20, 7298-7302.

(13) Lee, K. Y.; Peters, M. C.; Anderson, K. W.; Mooney, D. J. Nature 2000, 408, 998-1000.

(14) Lee, K. Y.; Peters, M. C.; Mooney, D. J. Adv. Mater. 2001, 13, 837-839.

(15) Fernandes, P. A. L.; Delcea, M.; Skirtach, A. G.; Möhwald, H.; Fery, A. Soft Matter 2010, 6, 1879-1883.

(16) Mertz, D.; Hemmerlé, J.; Mutterer, J.; Ollivier, S.; Voegel, J.-C.; Schaaf, P.; Lavalle, P. Nano Lett. 2007, 7, 657-662. 
SYNOPSIS TOC. Stretch-induced Reactive Release from a Layer-by-Layer Enzymatic Platform, C. Vogt, D. Mertz, J.-C. Voegel, P. Schaaf, P. Lavalle.

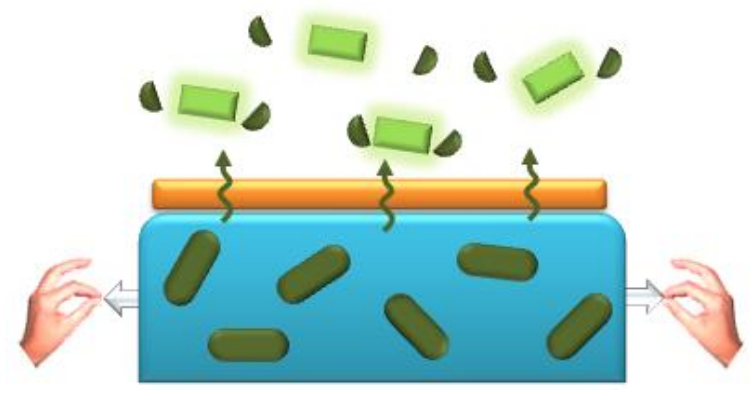

